\title{
Research on Cooperative Communication Technology to Improve Capacity and Reliability of Integrated Energy System
}

\author{
Gang Liu ${ }^{1 *}$, Huixian $\mathrm{Lu}^{1}$ \\ ${ }^{1}$ Smart Shine Microelectronics Technology Co. Ltd, Beijing, China
}

\begin{abstract}
The integrated energy system makes full use of renewable resources such as local photovoltaics, fully considers the difference and complementarity of load types in the region, and realizes the efficient, economic and clean utilization of regional energy from the dimensions of time and space. The existing communication system of the integrated energy system has become a constraint on business development. First, the communication rate is low and the system capacity is limited. After the terminal collects various energy information, a large amount of data needs to be uploaded, which puts huge pressure on the communication bandwidth. The second is poor reliability and high operation and maintenance costs. The current communication technology is single, equipment and networking costs are high. Aiming at the abovementioned pain points, an opportunistic relay cooperation technology and scheme based on signaling measurement is proposed. Theoretical analysis and simulation results show that the above scheme can improve the performance of the cooperative diversity system while reducing conflicts.
\end{abstract}

\section{Introduction}

The physical carrier of integrated energy and energy efficiency services is the integrated energy system, that is, making full use of local renewable resource such as photovoltaics, solar heat, and heat pumps, and fully considering the difference and complementarity of different load types in the region, from the terminal cold, heat, electricity load demand to power supply side, and realize the system of efficient, economical and clean utilization of regional energy in different time and space scales. Existing sensing equipment and information exchange technology have become constraints on the development of integrated energy service business. First, the communication rate is low and the system capacity is limited. After the terminal collects various energy information, a large amount of data needs to be uploaded, which puts huge pressure on the communication bandwidth. The second is poor reliability and high operation and maintenance costs. The current communication technology of integrated energy system is single, equipment and networking costs are high.

The mobile Internet and the Internet of Things realize information interaction between people, people and things, and things and things. Under the influence of factors such as complex terrain and electromagnetic interference, the wireless channel environment faces challenges of multiple types of fading. When the multipath fading effect is very strong, it will seriously affect the transmission of wireless signals and restrict the further development of wireless communication networks. In order to adapt to changes in the living environment, the field of wireless communication technology is actively exploring the adaptability of various new technologies to wireless networks. In addition to multi-carrier modulation, smart antennas, and adaptive transmission, diversity technology is also an important topic in wireless communication that resists fading to improve transmission reliability.

The methods to realize diversity include time diversity, frequency diversity and space diversity. Space diversity comes from the MIMO system [1], which is achieved by installing multiple antennas at the transmitter and receiver. Multiple antennas must be separated by a certain distance to ensure the independence of the channel. The signal copy arrives at the receiving end in the form of spatial redundancy, and the receiving end combines and decodes the received signals according to a certain combination criterion. However, in a wireless ad hoc network that is easily deployed, equipping each terminal with multiple antennas will not only increase the cost of the terminal, but also increase the complexity of its hardware and the power consumption of the whole device.

In order to overcome the limitations of multiple antennas, A. Sendonaris et al. proposed the concept of cooperative diversity [2]. The basic idea is to equip a mobile terminal with one or more partners, and obtain spatial diversity gain by making full use of the partners' spatial channels. Cooperative diversity can improve performance by the physical layer, but if the upper layer protocol is not properly designed, the advantages of the physical layer cannot be used to improve network performance. The cooperative MAC protocol [3-8] that matches the physical layer needs to consider how to choose cooperative nodes, how to avoid multiple access conflicts, and how to solve the problems of hidden

*e-mail: liugang2@sgitg.sgcc.com.cn 
terminals and exposed terminals in cooperative scenarios. In addition, synchronization, resource allocation and power allocation between cooperative nodes are also issues that need to be considered in the cooperative MAC protocol.

This article proposes an opportunistic relay cooperation scheme based on signalling measurement, which aims to solve the problem of cooperative transmission in synchronous ad hoc network systems. It reduces the signalling overhead of cooperative reservations and improves channel utilization by means of single reservation multiple multiplexing. The article first gives an overview of the cooperative access protocol, then proposes a protocol scheme based on signalling measurement, and conducts theoretical performance analysis and simulation verification on the relay strategy adopted by the protocol, and finally gives a summary.

\section{Cooperative access protocol}

The cooperative MAC protocol improves the diversity reception performance of the receiver by selecting a suitable intermediate node as the cooperative relay, and through the two-hop cooperative transmission of the relay node. According to the different of selection methods and transmission modes of cooperative relay nodes, cooperative MAC can be divided into three types: sender pre-selected cooperation, opportunistic cooperation, and coded cooperation protocol.

In the sender pre-selected collaboration, when a node needs to send a data packet, it selects the best collaboration node from the collaboration information table and sends a collaboration invitation. The typical CoopMAC protocol [5] maintains a collaboration table (CoopTable), in which the destination node, collaboration node, transmission rate, update time, and failure times are recorded in the table. The collaboration node meeting the following formula will be recorded in the CoopTable of the source node

$$
\frac{1}{R_{s h}}+\frac{1}{R_{h d}}<\frac{1}{R_{s d}}
$$

In the formula, $R_{s h}, R_{h d}, R_{s d}$ are the rates between the source node and the cooperative node, between the cooperative node and the destination node, and between the source node and the destination node, respectively. If the node has a packet to send, it searches for the cooperative node with the shortest transmission time in CoopTable and sends CoopRTS, which contains information such as cooperative node ID, $R_{\text {sh }}, R_{h d}$, etc. The cooperative node sends HTS when $R_{\text {sh }}$ and $R_{\text {hd }}$ are still valid, and the destination node waits for the corresponding HTS, and then reply to CTS When it receives CoopRTS. The source node successfully receives the CTS and sends it to the cooperating node at the rate $\mathrm{R}_{\text {sh }}$, and then a cooperating node sends it to the destination node at the rate $\mathrm{R}_{\text {hd. }}$.

The CARD protocol [6] estimates or obtains rate information by listening to RTS/CTS/DATA, and maintains a relay-weights table (relay-weights), which records the MAC address and weight value of the relay node, which can be calculated for the save time. In the CARD protocol, the relay node is designated to send
RCTS to participate in cooperation after the RTS/CTS has successfully interacted.

The opportunistic cooperation protocol refers to the potential cooperative nodes to listen to the transmission of node pairs, judge whether they can provide cooperation through a certain judgment algorithm, and participate in cooperative transmission if possible. The typical CRABR protocol [7] can estimate the channel state with the transceiver node by listening to RTS/CTS, and can extract the transmission rate between the transceiver nodes from the CTS. The node can obtain the rate supported by each link according to the channel state information. If the cooperative relay can save time, the node regards itself as a candidate cooperative node. The candidate cooperative node adopts the $\mathrm{p}$ persistence back-off strategy to send RTR (Ready to Relay) messages in $\mathrm{N}$ time slots, the greater the probability $\mathrm{p}$ that the candidate node that can achieve a higher transmission rate persists in access. After the data transmission is completed, the receiving node uses the ACK message to cancel unnecessary channel reservations, and informs neighbouring nodes that the channel is idle.

In order to maximize the independence of the cooperation path, the coding cooperation technology is often used, and the channel coding technology is applied to the cooperation diversity system, and the coding symbols are reorganized between users to obtain better diversity gain. Coding cooperation (CODE) protocol [8] introduces network coding or space-time coding, and the cooperative node encodes the data and sends it again, which can obtain a certain degree of multiplexing gain on the basis of cooperative diversity.

The existing cooperative MAC protocol usually uses competition and cooperation to obtain the channel usage rights, and select appropriate relay nodes to participate in cooperative transmission. The disadvantage is that signalling interaction and channel competition are required before each transmission, which seriously reduces the channel Utilization rate. At the same time, the competitive and cooperative MAC protocol cannot be adapted to the synchronous ad hoc network based on time division multiple access (TDMA).

\section{Relay cooperative access protocol based on signalling measurement}

Based on the TDMA time frame structure of the synchronous ad hoc network, this paper provides a multinode cooperative access protocol using dynamic channel reservation mechanism. The protocol periodically exchanges time slot status information between two hop neighbouring nodes. When the node has information to send through $\mathrm{RTC} / \mathrm{DTC} / \mathrm{CTC}$ signalling interaction to request cooperation and avoid multiple access conflicts, the intermediate node measures the reference signal in the signalling, obtain the channel state from itself to the source node and destination node, and if it meets the preferred index of the relay node, then participate in cooperative transmission. In this process, if the relay node has no data transmission requirements, its cooperative response signal (CTC) can be multiplexed in multiple frames to 
continuously participate in the cooperative transmission of source node information.

\subsection{Frame structure design}

Figure 1 is a time frame structure diagram of a synchronous ad hoc network. Time frame (frame) is the basic unit of time slot resource scheduling, which is composed of several time slots (slot), and several time frames form a super-frame, which is the basic of network synchronization and time slot state maintenance time unit. In order to respond to the dynamic allocation of time slot resources, each time frame is divided into 3 phases: synchronization phase, reservation phase and data transmission phase, which are respectively used for time slot resource synchronization maintenance, time slot resource reservation and data transmission.

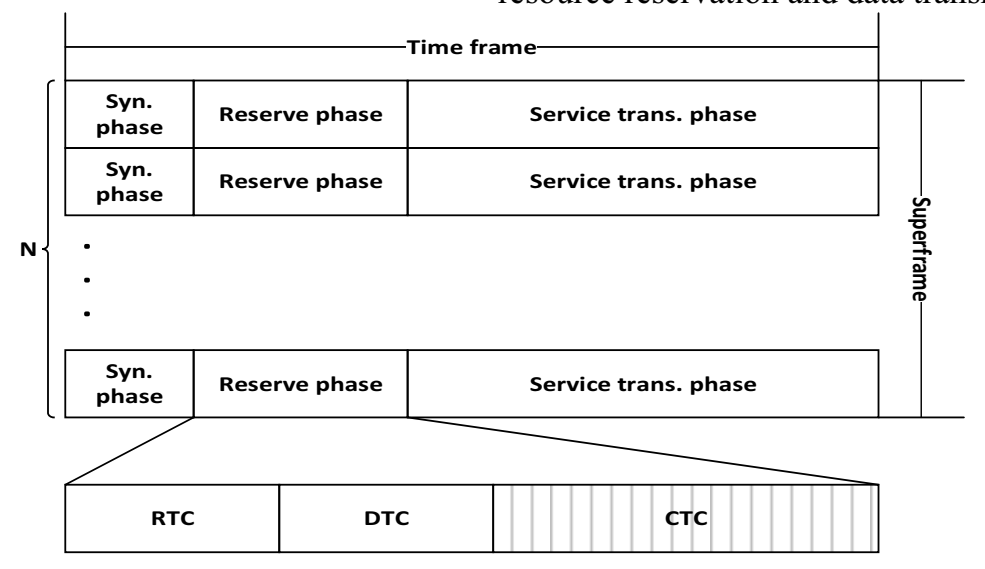

Figure 1. Time frame structure of synchronous ad hoc network

In the synchronization phase, each node periodically broadcasts the time slot status occupancy information of itself and the 1-hop neighbouring node. After its neighbouring node receives the time slot status occupancy information, it maintains the time slot status table of the 2hop neighbouring node. The time slot status occupancy table of the 2-hop neighbouring node can avoid the hidden terminal and exposed terminal problems in the distributed network to the greatest extent.

In the reservation phase, the node performs time slot reservation and cooperative relay node selection through signalling interaction. The source node sends Request-ToCooperate (RTC) in the subframe of request for cooperation RTC, and the destination node that receives the request for cooperation signalling returns a destination response (Destination-To-Cooperate, DTC) to the source node, ready for receiving. In the reservation phase, the intermediate node that listens to the signalling interaction between the source node and the destination node at the same time, measures the reference signal in the RTC and DTC signalling, and obtains the channel quality between the node and the source node, the node and the destination node (Channel State Indication, CSI). If the channel quality meets the criteria for participating in the coordinated transmission, the coordinate response signalling (Confirm-To-Cooperate, CTC) is sent to notify the source node and the destination node to prepare for the coordinated transmission.

In order to reduce the collision probability of synchronization response in the coordinated response signalling (CTC) of multiple cooperating nodes, the CTC response subframe is divided into multiple mini-slots, and time-slotted competition is used for contention multiplexing. Typically, the time-slotted competition mode can use time-slotted ALOHA and p-persistent timeslotted CSMA.

In the data transmission stage, the coordinated transmission adopts a two-slot coordinated transmission method. The source node sends the data packet directly to the destination node and the cooperative relay node in the first time slot. The relay node decodes in the first time slot and forwards it in the second time slot. The destination node combines and decodes the received data in the second time slot, thereby improving the signal-to-noise ratio and achieving better performance.

\subsection{Cooperative channel reservation method}

Figure 2 is a flowchart of a cooperative channel reservation method. When the source node receives a data transmission request, it sends a cooperation request (RTC) to the destination node and the intermediate node. The destination node returns a destination response (DTC) to the source node. The node located between the source node and the destination node performs signal measurement on the reference signal in the RTC and DTC signalling to obtain the channel quality from the node to the source node and the destination node. If the relay selection criterion is met, it will participate in decoding forwarding and cooperative transmission. The cooperative channel reservation signalling can reserve idle time slot resources of multiple time frames according to the time slot status table, and can maintain cooperative transmission until data transmission is completed. If the channel quality changes and the relay node changes, the local cooperation table is updated and the coordinated transmission continues. 


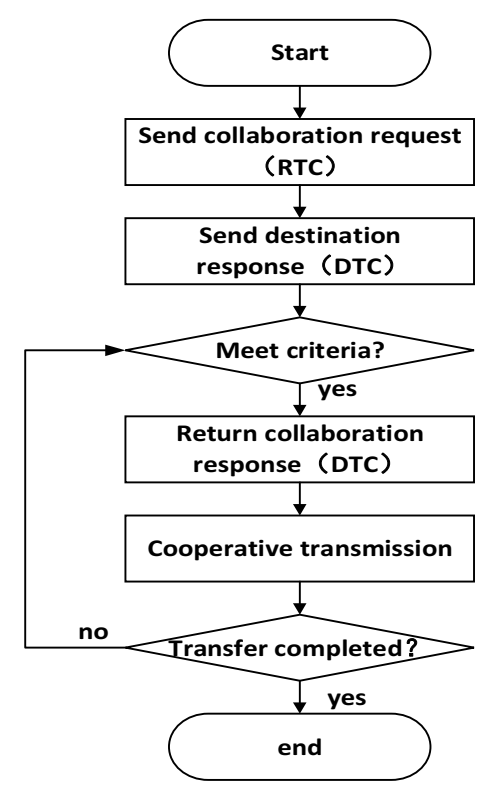

Figure 2. Cooperative channel reservation method flow

\subsection{Cooperative Relay Strategy}

In the face of multiple candidate relays, how to choose a suitable cooperative relay node is a key issue of the cooperative MAC protocol [9-10]. The channel quality, remaining energy, and load level of the cooperative node will directly affect the cooperation gain. Improper selection is likely to cause performance degradation.

In order to obtain effective diversity gain, this paper presents two typical cooperative relay selection strategies: opportunistic relay strategy and optimal relay strategy. Both of these two strategies use Channel State Indication (CSI) as the preferred criterion for the relay node. The opportunistic relay strategy adopts the threshold signal-tonoise ratio criterion. When the instantaneous signal-tonoise ratio of the relay node exceeds the minimum threshold required for correct decoding, it participates in decoding and forwarding. The opportunistic relay strategy's optimal criterion is as shown in equation (2).

$$
\min \left(\gamma_{s i}, \gamma_{i d}\right) \geq \gamma_{t h}
$$

Where $\gamma_{s i}, \gamma_{i d}$ are the instantaneous signal-tonoise ratios from the relay node $i$ to the source node and the destination node.

The optimal relay strategy adopts the maximum signal-to-noise ratio criterion, and only the node with the largest signal-to-noise ratio in the set of alternate relay nodes is decoded and forwarded, and other nodes do not participate. The optimization criterion of the optimal relay strategy is shown in formula (3), which $\gamma_{i}$ is the mean signal-to-noise ratio of relay node $i$, and $\mathrm{C}$ is the set of potential relay nodes.

$$
\gamma_{i}=\frac{1}{2}\left(\gamma_{s i}+\gamma_{i d}\right)=\max \left\{\gamma_{j}\right\}, j \in C
$$

The opportunistic relay strategy has multiple relay nodes participating in decoding and forwarding, while the optimal relay strategy has only one relay node with the best channel quality participating in forwarding. According to the capacity formula of virtual multi-antenna system (vMIMO) [11], the cooperative channel capacity of opportunistic relay strategy will be greater than that of optimal relay strategy. However, considering the realization of the cooperative communication system, the optimal relay strategy only involves the decoding and forwarding of one node, which is superior to the opportunistic relay strategy in terms of synchronization processing and energy consumption.

\section{Theoretical analysis and simulation evaluation}

\subsection{Rayleigh channel model}

Outage Probability is an important performance indicator to measure the cooperative relay system. It is defined as the cumulative probability (CDF) of a link outage event when the channel capacity $\mathrm{C}_{\gamma}$ is less than the link rate $\mathrm{R}$. The expression is as follows:

$$
P_{\text {out }}=P\{l b(1+\gamma)<R\}=\int_{0}^{2^{R}-1} p(\gamma) d \gamma
$$

In the formula, $\mathrm{R}$ is the normalized (unit bandwidth) link rate, $\mathrm{lb}($.$) is a logarithmic function$ with a base of $2, \gamma$ is the instantaneous signal-to-noise ratio, and $\mathrm{p}(\gamma)$ is the probability density of the instantaneous signal-to-noise ratio. The outage probability Pout of a single link mainly depends on the probability distribution model and parameters of the channel fading.

In order to simplify the analysis, the Rayleigh fading model with rich multipath components is usually used to analyze the outage probability [1216], and its instantaneous signal-to-noise ratio obeys the negative exponential distribution

$$
p(\gamma)=\frac{1}{\Gamma} \exp \left(-\frac{1}{\Gamma}\right)
$$

In the formula, $\Gamma$ is the mean value of the negative exponential distribution, then the outage probability of a single link can be expressed as

$$
P_{\text {out }}^{1}=\int_{0}^{2^{R}-1} \frac{1}{\Gamma} \exp \left(-\frac{1}{\Gamma}\right) d \gamma=1-\exp \left(-\frac{2^{R}-1}{\Gamma}\right)
$$

Different from single link communication, the link capacity of cooperative communication system is mutual information including direct transmission and relay channels [12]

$$
I=\sum_{i \in D(S)} I_{i}=\frac{1}{m+1} \sum_{i \in D(s)} l b\left(1+S N R \cdot \gamma_{i}\right)
$$

In the formula, $\mathrm{m}$ is the number of time slots required for cooperative transmission by the relay node, SNR is the signal-to-noise ratio of the sender, and $\gamma_{i}$ is the signal-to-noise ratio of the relay channel. At this time, the relay probability of a multirelay cooperative communication system using a 
decode-and-forward (DF) strategy [12] can be expressed as

$$
P_{\text {out }}^{c}=P(I<R)=\sum_{D(S) \in C} P(I<R \mid D(S)) P(D(S))
$$

In the formula, set $\mathrm{C}$ represents all relay combinations, $D(S)$ represents the set of relay nodes that can correctly decode the source node message, and $\mathrm{P}(\mathrm{I}<\mathrm{R} \mid \mathrm{D}(\mathrm{S}))$ represents the conditional interruption probability of the link capacity of the selected relay node set is less than the required link rate.

In the cooperative relay communication model described herein, the source node $S$ and the destination node $\mathrm{D}$ exchange channel state information through cooperation request (RTC) and cooperation response (CTC) signalling. According to different optimization strategies, several $(\mathrm{k})$ relay nodes R will be selected. Different numbers of relay nodes and different relay selection strategies will have an important impact on the performance of the cooperative relay system. Two typical relay selection strategies are given below, following the performance analysis.

\subsection{Opportunistic relay strategy}

The opportunistic relay strategy is also called the threshold selection relay strategy, that is, all relay nodes that meet the decoding conditions use themselves as cooperative relays to participate in decoding and forwarding. According to the assumption of Rayleigh fading, the outage probability described in (8) can be analysed.

Hypothesis 1: The channel fading between all nodes (source node-relay node, relay nodedestination node) is independent and identically distributed (i.i.d) and all obey negative exponential distribution.

Hypothesis 2: In order to simplify the analysis, the negative exponential distribution parameters of all nodes are consistent, and the mean signal-to-noise ratio is $\Gamma$.

The probability that the number of relay nodes satisfying the decoding condition $|\mathrm{D}(\mathrm{S})|$ is $\mathrm{k}$

$$
\begin{gathered}
P(|D(S)|=k)=\left(\begin{array}{l}
c \\
k
\end{array}\right) P(x>r)^{k} P(x<r)^{c-k}= \\
\left(\begin{array}{l}
c \\
k
\end{array}\right)\left(\int_{r}^{+\infty} \frac{1}{\Gamma} \exp \left(-\frac{x}{\Gamma}\right) d x\right)^{c-k}=\left(\begin{array}{l}
c \\
k
\end{array}\right)\left(e^{-\frac{\gamma}{\Gamma}}\right)^{k} \\
\left.e^{-\frac{\gamma}{\Gamma}}\right)^{c-k}
\end{gathered}
$$

Under the condition that all nodes participate in decoding and forwarding, the conditional interruption probability of the set of relay nodes $\mathrm{D}(\mathrm{S})$ whose link capacity is less than the required link rate is

$$
P\left(I_{\text {sum }}<R|| D(S) \mid=k\right)=P\left(\sum_{i=0}^{k} x_{i}<\frac{2^{(m+1) R}-1}{S N R}\right)
$$

For the independent and identically distributed (i.i.d) exponential random variable xi that obeys the parameter $1 / \Gamma$, the moment generating function of the integral variable $x_{\text {sum }}=\sum_{i=0}^{k} x_{i}$ is

$$
M_{\text {sum }}(\mathrm{s})=\left(\frac{1}{\Gamma \mathrm{s}+1}\right)^{k+1}
$$

Applying the inverse Laplace transform (IVT) to the above formula, the cumulative distribution function (CDF) of $x_{\text {sum }}$ is obtained as

$$
F_{\text {sum }}(x)=1-e^{-\frac{x}{\Gamma}} \sum_{i=0}^{k} \frac{\left(\frac{x}{\Gamma}\right)^{i}}{i !}
$$

Substituting the upper limit of integral of equation (8) into the above equation, it can be got

$$
\begin{gathered}
P\left(I_{\text {sum }}<R|| D(S) \mid=k\right)=F_{\text {sum }}\left(\gamma_{0}\right)=1- \\
e^{-\frac{x}{\Gamma}} \sum_{i=0}^{k} \frac{\left(\frac{x}{\Gamma}\right)^{i}}{i !}
\end{gathered}
$$

Combining equations (9) and (13), the outage probability of the cooperative relay system under the opportunistic relay strategy is

$$
\begin{gathered}
P_{\text {out }}^{c}=P\left(I_{\text {sum }}<R\right)=\sum_{k=0}^{c}\left(\begin{array}{l}
c \\
k
\end{array}\right)\left(e^{-\frac{\gamma_{0}}{\Gamma}}\right)^{k}(1- \\
\left.e^{-\frac{\gamma_{0}}{\Gamma}}\right)^{c-k}\left(1-e^{-\frac{\gamma}{\Gamma}} \sum_{i=0}^{k} \frac{\gamma_{0}}{\Gamma \cdot \cdot i !}\right)
\end{gathered}
$$

In the formula, $c$ is the total number of relay nodes, $k$ is the number of relay nodes participating in decoding and forwarding, $m$ is the number of cooperative time slots, $\gamma_{0}=\frac{2^{(m+1) R}-1}{S N R}$ is the effective information rate, and $\Gamma$ is the average signal-to-noise ratio of each relay channel.

\subsection{Optimal relay strategy Opportunistic relay strategy}

The optimal relay strategy refers to selecting the optimal relay node in the relay node set as the instantaneous relay to participate in decoding and forwarding. The most commonly used optimization criterion is the maximum signal-to-noise ratio $\left(\gamma_{\max }\right)$ criterion. At this time, the number of relay nodes is 1.

Under the condition that the probability distribution and parameters of the relay channel are consistent with the aforementioned assumptions, the conditional outage probability of the optimal relay strategy is

$$
P_{\text {out }}^{c}=\sum_{k=1}^{c} P\left(I_{\text {max }}<R \mid D(S)\right) P(D(S))
$$

In the formula, $I_{\max }$ represents the link capacity with the largest signal-to-noise ratio in the relay channel. The conditional outage probability of this link is 


$$
P\left(I_{\max }<R \mid D(S)\right)=P\left(x_{\max }<\frac{2^{2 R}-1}{S N R}\right)
$$

The cumulative distribution function for finding the maximum value $x_{\max }$ among $\mathrm{k}$ independent and identically distributed (i.i.d) random variables $x_{i}(i \in$ $(1, \ldots, \mathrm{k}))$ is

$$
F\left(x_{\max }\right)=\prod_{i=1}^{k} F_{i}(x)=\left(1-e^{-\frac{\gamma_{0}}{\Gamma}}\right)^{k}
$$

In the formula, $\gamma_{0}=\frac{2^{2 R}-1}{S N R}$ is the effective information rate, and $\Gamma$ is the average signal-to-noise ratio of each relay channel.

Combining equations (9) and (17), the outage probability of the cooperative relay communication system under the optimal relay strategy is

$$
\begin{gathered}
P_{\text {out }}^{c}=P\left(I_{\max }<R\right)=\sum_{k=0}^{c}\left(\begin{array}{l}
c \\
k
\end{array}\right)\left(e^{-\frac{\gamma_{0}}{\Gamma}}\right)^{k}(1- \\
\left.e^{-\frac{\gamma_{0}}{\Gamma}}\right)^{c-k}\left(1-e^{-\frac{\gamma}{\Gamma}}\right)^{k}
\end{gathered}
$$

In the formula, $c$ is the total number of relay nodes, $k$ is the number of relay nodes whose instantaneous signal-to-noise ratio is greater than the threshold, the number of cooperative time slots is 1 .

\subsection{Simulation evaluation}

The Monte Carlo simulation of the cooperative relay system is carried out by MATLAB tool, and the performance of the probability of interruption $\left(\mathrm{P}_{\text {out }}\right)$ of the above-mentioned opportunistic relay strategy and the optimal relay strategy is verified.

Firstly, consider the influence of the signal-tonoise ratio (SNR) of the transmitter on the probability of interruption $\left(\mathrm{P}_{\text {out }}\right)$. Choose the number of cooperative time slots $\mathrm{m}=1$, and the average signal-to-noise ratio of each relay channel $\bar{\gamma}_{\mathrm{s} r_{i}}=$ $\bar{\gamma}_{r_{i} d}=\Gamma=1 d B$. Figure 3 shows the comparison of Monte Carlo simulation and performance analysis results of two relay strategies under symmetrical channel conditions with 8PSK modulation and Turbo coding rate $1 / 2$.

It can be seen from Figure 3 that the outage probability Pout shows a downward trend as the SNR of the sender increases, and as the number of relay nodes c increases, the outage probability decreases more obviously, indicating that the increase in the number of relay nodes helps increase the reliability of cooperative communication. The comparison results of the opportunistic relay scheme (Scheme1) and the optimal relay transmission scheme (Scheme2) show that the outage probability performance of scheme 1 is better than scheme 2 , and as the signalto-noise ratio of the sender increases, its diversity gain is also more obvious.

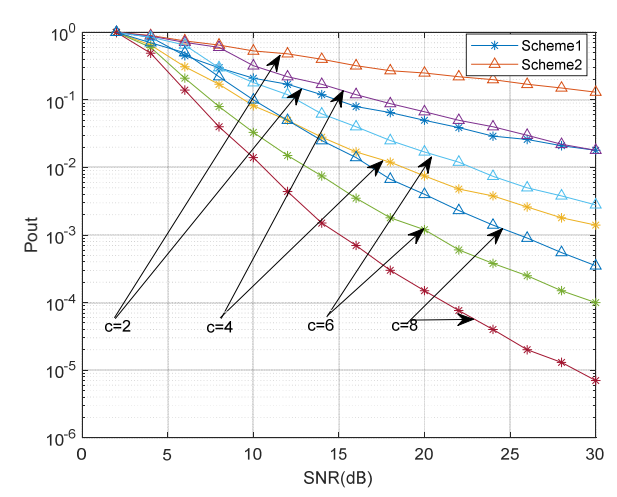

Figure 3. Pout-SNR performance curve

The comparison result of the two relay transmission schemes shows that the reliability of the opportunistic relay scheme in which all relay nodes participate in decoding and forwarding is better than the optimal relay scheme in which only one node participates in decoding and forwarding. However, the energy efficiency of the optimal relay scheme and the possibility of causing multiple access conflicts are lower than that of opportunistic relay, the corresponding MAC layer transmission control mechanism is much simplified.

Secondly, consider the influence of the signal-to-noise ratio $(\Gamma)$ of the relay channel on the probability of outage $\left(\mathrm{P}_{\text {out }}\right)$. Select the number of cooperative time slots $\mathrm{m}$ as 1 , and the signal-to-noise ratio SNR of the transmitter as $10 \mathrm{~dB}$. Figure 4 shows the comparison of Monte Carlo simulation and outage probability performance analysis results of two relay strategies under symmetrical channel conditions with 8PSK modulation and Turbo coding rate $1 / 2$.

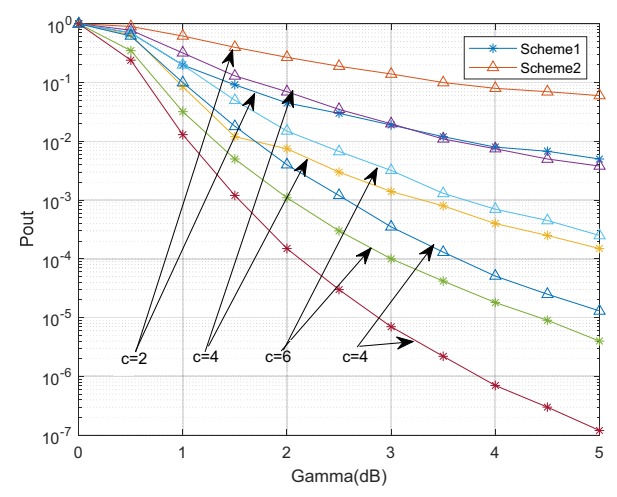

Figure 4. $P_{\text {out }} \Gamma$ performance curve

It can be seen from figure 4 that the outage probability $\mathrm{P}_{\text {out }}$ decreases with the increase of the relay channel signalto-noise ratio $\Gamma$, indicating that the better the relay channel quality, the greater the diversity gain produced by cooperative diversity. And with the increase of the number of relay nodes $c$, the downtrend of outage probability becomes more obvious, indicating that increasing the number of relay nodes helps to increase the reliability of cooperative communication. At the same time, the comparison results of the simulation curves in figure 4 can also show that the outage probability performance of scheme 1 is better than scheme 2, and as the signal-tonoise ratio of the sender increases, its diversity gain is 
more obvious.

Finally, consider the influence of the normalized link rate $(\mathrm{R})$ on the probability of outage $\left(\mathrm{P}_{\text {out }}\right)$. Choose the number of cooperative time slots $\mathrm{m}$ as 1 , the signal-tonoise ratio SNR of the transmitter as $10 \mathrm{~dB}$, and the average value of the signal-to-noise ratio of each relay channel $\gamma$. Figure 5 shows the comparison of Monte Carlo simulation and performance analysis results with different modulation orders (MCS).

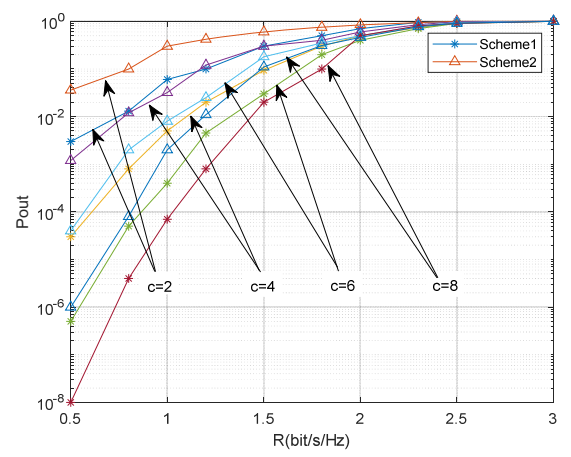

Figure 5. $\mathrm{P}_{\text {out }}-\mathrm{R}$ performance curve

It can be seen from figure 5 that the interruption probability $\mathrm{P}_{\text {out }}$ increases with the increase of the rate level (spectrum utilization). In the case of low rate transmission $(\mathrm{R}<0.8)$, the interruption probability can be as small as $10^{-}$ ${ }^{8}$, while in the case of high rate level $(\mathrm{R}>1.5)$, the maximum value of the outage probability approaches 1 , and the channel capacity of the cooperative relay can no longer meet the transmission rate requirement. The curve shown in figure 5 also shows that as the transmission rate decreases, the outage probability performance of the opportunistic relay scheme increases more significantly, and the outage probability performance of the opportunistic relay scheme is better than that of the optimal relay scheme.

The above simulation analysis results show that the outage probability performance of the opportunistic relay scheme is better than that of the optimal relay scheme. This is because the opportunistic relay scheme uses a threshold relay strategy, and all relay nodes whose signalto-noise ratio exceeds the threshold participate in decoding and forwarding, and the probability of link interruption is less than the optimal relay scheme where only one node participates in decoding and forwarding.

\section{Conclusion}

This paper studies the cooperative multiple access technology in the synchronous ad hoc network, proposes a time slot reservation method for multi-stream coordination, and gives two different relay node optimization strategies for opportunistic relay and optimal relay. Theoretical analysis and simulation results show that the outage probability performance of the opportunistic relay strategy is better than that of the optimal relay strategy.

If the physical layer adopts beamforming and power allocation strategies, other performance indicators of the aforementioned distributed access protocol, such as outage probability, bit error rate, throughput rate, require further simulation analysis. If the difficulty of terminal implementation is taken into consideration, the abovementioned protocol complexity, computational complexity, energy efficiency and other indicators also need to be further evaluated.

\section{Acknowledgments}

We would like to express our gratitude to Zhongxiao $\mathrm{Li}$ and Shouqiang Zhao who provided strong technical support for this article, and to Smart Shine Microelectronics LTD. who provided strong financial support for this article, and to the conference organizers who provided technical support for the publication of this article.

\section{References}

1. W.M. Jang. Diversity order and coding gain of general-order rectangular QAM in MIMO relay with TAS/MRC in Nakagami-fading [J]. IEEE Trans. on Vehicular Technology, 2014, 63(7): 3157-3166.

2. A. Sendonaris, E. Erkip, B. Aazhang. User cooperation diversity. Part I and II [J]. IEEE Trans. on Communications, 2003, 51(11): 1927-1948.

3. J. Feng, R. Zhang, et al. Cooperative medium access control based on spectrum leasing [J]. IEEE Trans. on Vehicular Technology, 2014, 63(1):297-307.

4. H. Qin, R. Zhang, et al. Distributed cooperative MAC for wireless networks based on network coding [C]. Wireless Communications and Networking Conference (WCNC), New Orleans, LA, 2015: 20502055.

5. P. Liu, Z. Tao, et al. CoopMAC: a cooperative MAC for wireless LANs [J]. IEEE Journal on Selected Areas in Communications, 2007, 25(2): 340-354.

6. S. Sayed, Y. Yang, H. Hu. CARD: cooperative access with relay's data for multi-rate wireless Local Area Networks [C]. IEEE International Conference on Communications (ICC'09), Dresden, Germany, 2009:1-6.

7. N. Li, N. Cheng, et al. Performance analysis of a cooperative MAC based on opportunistic relaying for ad hoc networks [C]. 2010 International Conference on Wireless Communications and Signal Processing (WCSP), Suzhou, China, 2010:1-6.

8. S. Kumar, H. Lim, H. Kim. Hierarchical MAC protocol with multi-channel allocation for enhancing IEEE 802.11 ah relay networks [C]. 2015 International Conference on Wireless Communications and Mobile Computing, Dubrovnik, Croatia, 2015: 1458-1463.

9. N. Marchenko, C. Bettstetter. Cooperative ARQ with relay selection: an analytical framework using semiMarkov processes [J]. IEEE Trans. on Vehicular Technology, 2014,63(1): 178-190.

10. E. Molina, J. Matias, et al. Managing path diversity in layer 2 critical networks by using OpenFlow [C]. 
IEEE Network and Service Management (CNSM), Barcelona, Spain. 2015: 394-397.

11. E. Telatar. Capacity of multi-antenna Gaussian channels [J]. AT\&T Bell Technical Memorandum, 1995.

12. J.N. Laneman and G.W. Wornell. Distributed spacetime-coded protocols for exploiting cooperative diversity in wireless networks[J]. IEEE Trans. Inform Theory, 2003, 49: 2415-2425.

13. Y. Zhao, R. ADVE, T.J. LIM. Outage probability at arbitrary SNR with cooperative diversity[J]. IEEE Communication Letters, 2005, 9(8):700-702.

14. T.E. Hunter, S. Sanayei, A. Nosaratinia. Outage analysis of coded cooperation [J]. IEEE Trans. Information Theory, 2006, 52(2): 375-391.

15. S. Nadarajah. On outage probability of selection cooperation [C]. Wireless Pers Commun, 2013, 71:1331-1337.

16. G. Cheng, et al. Outage probability analysis of decode-and-forward relaying systems with energy harvesting [C]. 2018 4th International Conference on Big Data Computing and Communications (BIGCOM), 2018, 1: 28-33. 